\title{
Personalidad, estilos de aprendizaje y competencias digitales de estudiantes universitarios en modalidad remota por la pandemia COVID-19
}

\section{Personality, learning styles and digital competences of university students in remote learning for the COVID-19 pandemic}

HERNÁNDEZ-ROMERO, María I. ${ }^{1}$

\begin{abstract}
Resumen
Esta investigación cuantitativa se centra en dos variables personales con relación al nivel autopercibido de competencias digitales de 278 estudiantes universitarios. Los resultados emanados de un instrumento integrador mostraron que el nivel de competencia digital reportado fue entre intermedio y avanzado, quizá motivado por la pandemia. La extroversión correlacionó débilmente con dos áreas de competencias digitales, pero no con los estilos de aprendizaje. Interesantemente, la distribución entre estilos de aprendizaje y niveles de competencias sí mostró algunas tendencias a favor del estilo asimilador.
\end{abstract}

Palabras clave: personalidad; estilos de aprendizaje; competencias digitales; COVID-19

\begin{abstract}
This quantitative research focuses on two personal variables in relation to the self-perceived level of digital skills of 278 university students. The results emanating from an integrating instrument showed that the level of digital competence reported was between intermediate and advanced, perhaps motivated by the pandemic. Extraversion was weakly correlated with two areas of digital competencies, but not with learning styles. Interestingly, the distribution between learning styles and competence levels did show some tendencies in favor of the assimilating style.

Key words: personality; learning styles, digital competences; COVID-19
\end{abstract}

\section{Introducción}

En la última década se han realizado varias investigaciones sobre las competencias digitales de los estudiantes universitarios, principalmente para describir su grado de desarrollo motivado por el uso de las tecnologías de la información y comunicación (Guzmán Flores, 2008; Guzmán Flores y Gisbert Cervera (2008); Cabero et al, 2009; Vázquez-Cano, Reyes Vélez, Colmenares Zamora y López Meneses, 2017; Sánchez-Macías y Veytia-Bucheli, 2019; Marín, Hernández, Borges y Blanqueto, 2021). También en estos estudios se han incorporado variables como la edad, la carrera y el género para establecer diferencias y relaciones con las competencias digitales. Sin embargo, se parte de la premisa de que otras variables personales pudieran determinar el uso de las competencias

\footnotetext{
${ }^{1}$ Profesora-investigadora de Tiempo Completo. Universidad de Quintana Roo, México. Correo electrónico: isaherna@uqroo.edu.mx
} 
digitales, sobre todo en un contexto atípico ocasionado por la pandemia del COVID-19. Por lo tanto, el interés en la presente investigación es explorar la relación que pudiera existir entre dos variables dentro del ámbito de la psicología con respecto al grado de desarrollo de las competencias digitales en el estudiantado universitario.

Por un lado, se ha seleccionado la variable extroversión como un componente importante de la personalidad, cuyos rasgos distintivos han estado asociados al desempeño académico de los estudiantes. Por otro lado, los estilos de aprendizaje también han estado vinculados a aspectos clave de la educación tales como los diseños instruccionales, evaluación y contextos de aprendizaje. De allí proviene la motivación que invita a describir la personalidad y estilos de aprendizaje de los estudiantes con relación al grado de desarrollo de sus competencias digitales. A continuación, se reseñan sucintamente las tres variables de importancia en esta investigación.

\subsection{Extroversión como factor de la personalidad}

Han sido varios los esfuerzos por describir el constructo de la personalidad y las diferentes teorías para explicarlo que han emergido a lo largo de los años. Marín-Marín (2005) hace un breve recuento de estas teorías, iniciando por la definición de la personalidad, continuando con las teorías de los tipos y de los rasgos y finalizando con el Modelo de Personalidad de Eysenck. De esta manera, la personalidad se puede definir como:

"una organización más o menos estable y duradera del carácter, el temperamento, la inteligencia y el físico de una persona, que determina su adaptación única al entorno. El carácter denota el sistema más o menos estable y duradero de comportamiento conativo de una persona (voluntad); temperamento, su sistema más o menos estable de comportamiento afectivo (emoción); el intelecto, su sistema más o menos estable y final de comportamiento cognitivo, (inteligencia); físico, su sistema más o menos estable y final de configuración corporal y dotación neuroendocrina" (H. J. Eysenck, 1970, citado en Marín 2005, p. 16).

Esta definición ha sido una de las más influyentes en lo que se conoce como la perspectiva biológica de la personalidad, la cual se contrapone a las teorías situacionistas e interaccionistas, puesto que Eysenck consideraba que los seres humanos nacen con sistemas personales predeterminados que se activan a lo largo de la vida. Al igual que Marín-Marín (2005), para efectos de este estudio se ha adoptado el trabajo de Eysenck dado que su teoría ha sido respaldada por estudios experimentales y descriptivos que lograron explicar su Modelo PEN de la personalidad (psicotismo, extroversión y neuroticismo). De este modelo, ha llamado la atención el factor extroversión que ha sido estudiado ampliamente por su relación con el rendimiento escolar y con aspectos laborales y de convivencia. Por lo tanto, resulta necesario describir qué se entiende por extroversión y cómo se puede operacionalizar como una variable de importancia en la vida diaria.

Según H.J. Eysenck y S.B. Eysenck (1969), el factor extroversión, al igual que el psicotismo y el neuroticismo, debe verse como un continuo con dos extremos: introversión-extroversión; en otras palabras, una baja extroversión implica alto grado de introversión. Es por ello que Eysenck describe al típico individuo extrovertido como sociable, que le gustan las fiestas, que tiene muchos amigos, que necesita platicar con la gente, que le encantan las emociones y actuar de manera espontánea y que generalmente es impulsivo, entre otros rasgos. En contraste, el típico introvertido suele ser callado, retraído e introspectivo, reservado y distante pero no con sus amigos cercanos; le gustan los libros más que hacer amigos, tiende a planear las cosas y no le gusta el impulso del momento.

Una de las razones por basar este estudio en el trabajo de H. J. Eysenck $(1947,1967,1990)$ tiene que ver con los métodos estadísticos que utilizó como el análisis factorial con el que logró reducir cientos de variables para describir la personalidad a solo tres factores o dimensiones. La estructura jerárquica de esos tres factores ha ayudado a comprender cómo se integran las dimensiones de la personalidad; es decir, desde un nivel específico 
de respuesta, pasando por un nivel habitual hasta llegar a un nivel de rasgo personológico (sociabilidad, impulsividad, actividad, emotividad, etc.). Otra de las razones por la que se adopta la teoría de Eysenck estriba en la serie de instrumentos de medición de la personalidad en sus tres dimensiones, los cuales han sido validados a través de los años. Por ejemplo, uno de los primeros instrumentos fue el Inventario de Personalidad de Eysenck (EPI por sus siglas en inglés) y uno de sus últimos fue el Cuestionario de Personalidad de Eysenck (EPQ-R), cuya versión corta se utilizó en este estudio.

\subsection{Estilos de aprendizaje}

Ciertamente, la investigación sobre estilos de aprendizaje ha jugado un papel primordial en el campo de la educación y en el laboral. Como segundo constructo, se ha incorporado en esta investigación el concepto de estilo de aprendizaje, el cual es una aportación importante de la psicología cognitiva. Al igual que en otras teorías, no existe una definición de estilos de aprendizaje ampliamente aceptada, dado que cada modelo propuesto conlleva su propia descripción del constructo. Para Keefe $(1979$, p. 4) los estilos de aprendizaje son "comportamientos cognitivos, afectivos y fisiológicos característicos que sirven como indicadores relativamente estables de cómo los alumnos perciben, interactúan y responden al entorno de aprendizaje". Esta definición parece englobar algunos rasgos de la personalidad desde el punto de vista biológico y que son parte de las diferencias individuales.

Uno de los principales impulsores de los estilos de aprendizaje ha sido D. Kolb desde los años setenta, quien propuso su teoría del aprendizaje experiencial. D. Kolb y A. Kolb (2013) definen los estilos de aprendizaje como "las formas únicas en que los individuos escalan el ciclo de instrucción a través de su preferencia por los cuatro modos aprendizaje" (p. 9). Estos cuatro modos de aprendizaje parten de dos premisas: las formas que los individuos perciben la información y las maneras en que la procesan; en otras palabras, cómo se entiende la experiencia y cómo se transforma. De allí proponen el ciclo de aprendizaje experiencial que incluye la experiencia concreta (EC), la observación reflexiva (OR), la conceptualización abstracta (CA y la experimentación activa (EA). Interesantemente, estas cuatro formas de percibir y transformar la información dan cabida a los cuatro estilos de aprendizaje propuestos por Kolb. La Figura 1 es una adaptación de este ciclo de aprendizaje de Kolb.

Figura 1

El ciclo de aprendizaje experiencial de Kolb

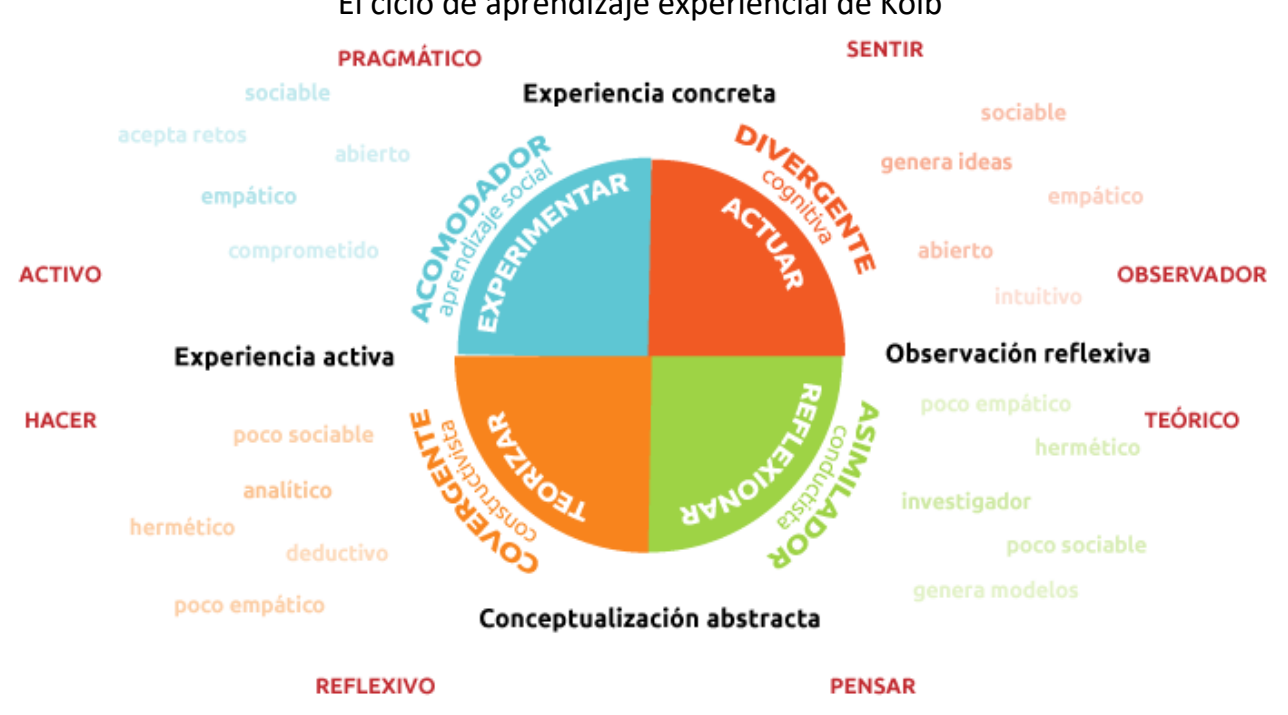

Fuente: Vergara, C. (s.f.) La teoría de los estilos de aprendizaje de Kolb. Actualidad en Psicología. https://www.actualidadenpsicologia.com/la-teoria-de-los-estilos-de-aprendizaje-de-kolb/ 
Se reitera que las dualidades entre los cuatro modos de experiencia dan pie a los cuatro estilos de aprendizajes identificados por Kolb (2015, pp. 114-115), quien los resume así:

1. El estilo de aprendizaje convergente depende primordialmente de las habilidades dominantes de aprendizaje de la conceptualización abstracta (CA) y la experimentación activa (EA). La fortaleza mayor radica en la resolución de problemas, la toma de decisiones y la aplicación práctica de las ideas. Hay preferencia por las actividades y problemas técnicos más que en las situaciones sociales e interpersonales. Las personas convergentes se contienen en la expresión de su emoción.

2. El estilo de aprendizaje divergente tiene las fortalezas de aprendizaje opuesta al estilo convergente, ya que se enfatiza la experiencia concreta (EC) y la observación reflexiva (OR). El punto más fuerte de esta orientación radica en la habilidad imaginativa y la conciencia del significado y los valores. La habilidad primaria adaptativa en este estilo es el poder ver situaciones concretas desde varias perspectivas. Las personas divergentes están interesadas en las personas y tienden a ser imaginativas y orientadas a los sentimientos.

3. En el estilo de aprendizaje de asimilación las habilidades de aprendizaje dominantes son la conceptualización abstracta (CA) y la observación reflexiva (OR). La fortaleza mayor recae en el razonamiento inductivo y la habilidad para crear modelos teóricos, así como en asimilar observaciones disparejas en explicaciones integradas. Al igual que en el estilo convergente, hay poco interés en las personas y mayor atención a las ideas y conceptos abstractos. De importancia es que teoría sea sólida y precisa.

4. El estilo de aprendizaje de acomodación resulta lo opuesto a las fortalezas de la asimilación ya que se prioriza la experiencia concreta $(\mathrm{EC})$ y la experimentación activa (EA). Los puntos más fuertes de esta orientación tienen que ver con hacer las cosas, llevar a cabo planes y tareas e involucrarse en nuevas experiencias. Se hace mucho énfasis adaptativo en la búsqueda de oportunidades, la toma de riesgos y la acción. Las personas con este estilo se interesan en la gente, pero algunas veces parecen impacientes y avasalladoras.

Al igual que Eysenck y su Modelo PEN de personalidad, Kolb (1984) ha desarrollado instrumentos validados psicométricamente para detectar los estilos de aprendizaje. Este instrumento se conoce como el Inventario de Estilos de Aprendizaje de Kolb, el cual ha recibido revisiones desde sus inicios en los años setenta. El inventario de Kolb es un instrumento de autopercepción y de acuerdo con su autor puede servir para propósitos de investigación, de aplicación educativa y laboral. Es un instrumento conciso y estructurado de 9 y 12 reactivos, en el que los usuarios pueden obtener resultados inmediatos y graficados. Los reactivos incluyen una situación de aprendizaje con cuatro opciones y los usuarios tiene que numerarlas del 1 al 4 según corresponda a su situación de aprendizaje. En resumen, el inventorio de Kolb resulta práctico de aplicar y evaluar.

\subsection{Competencia digital}

La variable dependiente en esta investigación es la competencia digital, concepto que ha evolucionado en los últimos años; es decir, desde tener un sentido instrumentalista hasta uno más integrador. Como en el caso de la definición de personalidad y estilos de aprendizaje, existen varias definiciones que reflejan esa evolución y dinamismo. Para efectos de esta investigación y por razones teórico-metodológicas, se adopta la definición establecida en el Marco de Competencia Digital Docente auspiciado (INTEF, 2017), la cual se deriva del Proyecto DigComp del Parlamento Europeo. 
"aquí preferimos referirnos a la competencia digital como el conjunto de conocimientos, habilidades y actitudes necesarias hoy en día para ser funcional en un entorno digital. Por tanto, además de los cambios que introducen las nuevas tecnologías en la alfabetización de la lectura y escritura, podemos argumentar que la competencia digital requiere un conjunto nuevo de habilidades, conocimientos y actitudes." (INTEF, 2017, p. 12)

Como se puede observar, esta definición de competencia digital incorpora los tres elementos importantes de toda competencia: conocimientos, habilidades y actitudes indispensables para que el ciudadano del Siglo XXI pueda participar activamente en ambientes digitales. En otras palabras, se pueden tener los conocimientos, pero no las habilidades; se pueden tener las habilidades, pero no las actitudes hacia la tecnología o hacia la sociedad misma.

Independientemente de los diferentes marcos existentes como son los de la UNESCO, la OCDE, y el ISTE (Marco de la Sociedad Internacional de Tecnología en Educación), el Marco de Competencia Digital Docente (INTEF 2017, p. 2) propone cinco grandes áreas competenciales que se enlistan a continuación:

\section{Área 1. Información y alfabetización informacional}

1.1. Navegación, búsqueda y filtrado de información, datos y contenidos digitales

1.2. Evaluación de información, datos y contenidos digitales

1.3. Almacenamiento y recuperación de información, datos y contenidos digitales

\section{Área 2. Comunicación y colaboración}

2.1. Interacción mediante las tecnologías digitales

2.2. Compartir información y contenidos digitales

2.3. Participación ciudadana en línea

2.4. Colaboración mediante canales digitales

2.5. Netiqueta

2.6. Gestión de la identidad digital

\section{Área 3. Creación de contenido digital}

3.1. Desarrollo de contenidos digitales

3.2. Integración y reelaboración de contenidos digitales

3.3. Derechos de autor y licencias

3.4. Programación

\section{Área 4. Seguridad}

4.1. Protección de dispositivos

4.2. Protección de datos personales e identidad digital

4.3. Protección de la salud

4.4. Protección del entorno

\section{Área 5. Resolución de problemas}

5.1. Resolución de problemas técnicos

5.2. Identificación de necesidades y respuestas tecnológicas

5.3. Innovación y uso de la tecnología digital de forma creativa

5.4. Identificación de lagunas en la competencia digital

Este marco contempla 21 competencias específicas alineadas jerárquicamente a las cinco áreas competenciales. Cada competencia contiene descriptores graduados a seis niveles de competencia ( $A 1$, A2, B1, B2, C1 y C2) y tres macro niveles: básico, intermedio y avanzado. Este grado de especificidad le proporciona al marco la solidez necesaria para ser un referente en la elaboración y medición de la competencia digital docente, que puede ser fácilmente extrapolable al contexto estudiantil. 
Habiendo reseñado brevemente las tres variables importantes de esta investigación, a continuación, se formulan tres preguntas de investigación.

1. ¿Cuáles son las competencias digitales reportadas con mayor y menor desarrollo por el estudiantado universitario durante la pandemia COVID-2019?

2. ¿Existe una relación entre la personalidad de los estudiantes universitarios y el grado de autopercepción de sus competencias digitales?

3. ¿Existe una relación entre los estilos de aprendizaje de los estudiantes universitarios y el grado autopercibido de sus competencias digitales?

La pregunta 1 se enfoca a describir la autopercepción que tienen los estudiantes universitarios con respecto a sus niveles de competencias digitales, ya sea por competencia específica o en las cinco categorías propuestas en el Marco Común de Competencia Digital Docente (INTEF, 2017). Las preguntas centrales 2 y 3 están encaminadas a examinar si el grado autopercibido de competencias digitales del estudiantado pudiera estar relacionado con su grado extroversión y sus estilos de aprendizaje.

\subsection{Revisión de la literatura}

En el contexto mexicano, el estudio de las competencias digitales de estudiantado universitario ha cobrado importancia en los últimos cinco años; en su mayoría enfocados a la frecuencia de uso de las herramientas TIC. Sin embargo, el interés en este tema se remonta al trabajo de Guzmán Flores (2008) y Guzmán Flores y Gisbert Cervera (2008), quienes investigaron el uso de las competencias TIC en una universidad pública del centro de México. La muestra estuvo compuesta de 326 estudiantes de licenciatura a quienes les aplicaron un cuestionario con reactivos en escala del 1 al 6 en cuatro áreas: alfabetización tecnológica, instrumentos de trabajo intelectual, tratamiento y difusión de la información y herramientas de comunicación. En general, las investigadoras encontraron que los estudiantes reportaron dominio por arriba de la media en la gestión de la información, el trabajo colaborativo y la comunicación TIC.

En otro estudio medular, Cabero et al (2009) llevaron a cabo un estudio sobre la alfabetización digital de 259 estudiantes de una universidad del noreste de México. Los autores utilizaron un cuestionario en una escala del 0 al 10 para medir la percepción de competencia digital, resultando una media global de 7.8 de la muestra. Asimismo, se encontraron medias arriba de 9 en el uso de motores de búsqueda y la comunicación a través de foros, mensajería instantánea y correo electrónico. Por el contrario, se reportaron medias relativamente bajas en la creación y modificación de bases de datos y el diseño de páginas web.

Por su parte, Vázquez-Cano, Reyes Vélez, Colmenares Zamora, y López Meneses (2017) realizaron un estudio cuantitativo descriptivo en el que participaron 465 estudiantes de una universidad ecuatoriana. Alineado al diseño de la investigación, los investigadores utilizaron el cuestionario "Competencias básicas digitales 2.0 de los estudiantes universitarios" COBADI (Marca registrada: 2970648), el cual consta de 23 reactivos agrupados en tres categorías: competencias TIC, competencias interpersonales TIC y herramientas de comunicación. Al igual que en los estudios anteriores, los resultados estriban en que las competencias más desarrolladas fueron las de gestión de la información y las de menor desarrollo fueron las competencias de creación y difusión de contenidos digitales.

En una investigación cuantitativa relativamente reciente, Sánchez-Macías y Veytia-Bucheli (2019) analizaron el nivel de competencia digital de 77 estudiantes de posgrado en áreas de administración, educación y socioformación. Como instrumento de medición, utilizaron un cuestionario de competencias digitales de 83 reactivos tipo Likert con una escala de cuatro niveles. Un análisis factorial confirmatorio arrojó ocho componentes principales; entre los cuales, las puntuaciones más altas se observaron en la gestión y 
procesamiento de la información y las habilidades para el trabajo creativo. Por otro lado, el componente menos desarrollado fue el entendimiento y concienciación sobre el papel de las TIC en la sociedad.

Cabe señalar que la presente investigación tiene como antecedente primario el estudio realizado por Marín, Hernández, Borges y Blanqueto (2021), quienes analizaron el grado de desarrollo de las competencias digitales de 678 estudiantes de una universidad pública del sureste mexicano. Como instrumento de medición, utilizaron un cuestionario de elaboración propia de 44 reactivos agrupados en cuatro categorías, teniendo como referencia el Marco Común de Competencia Digital Docente (INTEF, 2017). Los reactivos tuvieron una escala tipo Likert del 1 al 4 y fue aplicado en línea con visitas a las aulas en 2019. Entre los resultados de interés se destaca que el 78 $\%$ de los estudiantes se autopercibió con un nivel de competencia intermedio; un $12.5 \%$ en un nivel avanzado y el $8.5 \%$ como principiante. De igual manera, las áreas competenciales con mayor desarrollo fueron la comunicación y la gestión de información, mientras que el trabajo colaborativo y la creación de contenidos digitales resultaron con las medias más bajas.

En cuanto a la variable de personalidad asociada con las competencias digitales, se puede señalar que el panorama es distinto puesto que una revisión exhaustiva de la literatura no arrojó estudios previos en México. A nivel internacional se halló el trabajo de Nahyun y Hana (2011), quienes investigaron la relación de cincos rasgos de personalidad con la llamada competencia de información, la cual implica las estrategias de búsqueda y evaluación de la información en la red. Para la variable de personalidad utilizaron el Modelo de cinco factores de Costa y McCrae con cinco rasgos: neuroticismo, extroversión, apertura a la experiencia, amabilidad y responsabilidad, mientras que para la variable competencia de información usaron una sección de 10 reactivos de unos estándares de competencia informacional. Los participantes fueron estudiantes 188 estudiantes de una universidad de suroeste de Estados Unidos. Por demás interesante, con base en una regresión lineal, extroversión tuvo un valor predictivo de la competencia de información, aunque muy bajo $(B=.175)$.

Otra aproximación a estas dos variables es la investigación de Ahmed y Rasheed (2020), quienes examinaron la relación de cinco rasgos personológicos con las habilidades de alfabetización digital. Los participantes fueron 255 bibliotecarios de varias universidades de Pakistán. Se aplicó un cuestionario de personalidad que mide cinco rasgos, entre ellos, la extroversión, así como un cuestionario de alfabetización digital con cinco habilidades. Los investigadores encontraron que una relación positiva, pero débil, entre la extroversión y las cinco habilidades digitales: uso de la biblioteca, uso de la computadora, uso de herramientas digitales, gestión de la información y uso de bases de datos para la investigación. En otras palabras, los bibliotecarios con rasgos de extroversión parecen tener un mayor grado de desarrollo en sus habilidades digitales que los demás grupos de personalidad.

Con respecto al papel de los estilos de aprendizaje en el desarrollo de las competencias digitales, los estudios también son escasos. En la literatura, se han encontrado aproximaciones consistentes en investigar la relación entre los estilos de aprendizaje y el uso de las TIC como es el caso de Orellana, Bo, Belloch y Aliaga (2002). Los autores aplicaron el cuestionario Honey-Alonso que mide cuatro estilos de aprendizaje: activo, reflexivo, teórico y pragmático, así como un cuestionario de uso de la TIC (chat, correo electrónico, juegos y búsqueda de información. Entre los hallazgos, no se observó ninguna relación significativa entre ambas variables; es decir, el uso de las TIC resultó independiente de los estilos de aprendizaje de 169 estudiantes universitarios.

Otros estudios aproximados al tema se han realizado para examinar la relación entre los estilos de aprendizaje y el uso de la tecnología, aunque no necesariamente como competencias digitales. Por ejemplo, destaca la investigación de De Moya et al (2011), quienes intentaron describir el estilo de aprendizaje predominante en el uso de las TIC. Aunque no encontraron diferencias estadísticamente significativas, sí observaron algunas tendencias en la que los alumnos activos y reflexivos reportaron un mayor uso de las TIC. Por su parte, Romero, Salinas y Mortera (2010) aplicaron el Modelo de Kolb con estudiantes de modalidad virtual, cuyo estilo 
predominante fue el divergente. Sin embargo, los investigadores concluyeron que el estilo que se privilegia en la plataforma Moodle es el divergente y que un bajo porcentaje de los participantes resultó con ese estilo.

También en la literatura se encontró un estudio cuasi-experimental en el que se consideran los estilos de aprendizaje como variable interviniente y las competencias digitales, aunque con estudiantes de secundaria. Chapilliquén (2015) llevó a cabo la intervención en la Plataforma Edmodo con dos grupos de 80 y 82 estudiantes. Para la recolección de los datos, se utilizaron dos cuestionarios estructurados: uno de estilos de aprendizaje con ocho tendencias y el otro de competencias digitales con cinco dimensiones. Los resultados demostraron una distribución predominante de estudiantes con estilo sensorial y visual del grupo experimental con mayores niveles en el conocimiento y uso de las herramientas digitales, acceso y procesamiento de la información, la ética en el uso de la información digital y la comunicación de la información en diferentes formatos.

A manera de cierre de esta sección, se puede sugerir que la literatura sobre la competencia digital del estudiantado universitario es vasta comparada con los estudios en los que se incorporan las variables de personalidad y estilos de aprendizaje. Esto parece sugerir que existe poco interés en estos factores internos que bien pudieran explicar la variabilidad en el desarrollo de las competencias digitales de los estudiantes. En sí, los resultados de estas investigaciones son poco claros, quizá porque ha sido una temática escasamente explorada. Sin embargo, esta situación motivó aún más la realización de este estudio.

\section{Metodología}

La presente investigación tiene un diseño cuantitativo de corte descriptivo, cuyas variables independientes fueron la personalidad y los estilos de aprendizaje, mientras que las competencias digitales fueron la variable dependiente. En este apartado se describen los participantes de la investigación, los instrumentos y procedimientos, así como el análisis de los datos.

\subsection{Participantes}

Los participantes del estudio fueron 278 estudiantes de dos universidades públicas de México. De la muestra por conveniencia, el 65.8 \% fueron del género femenino y el 34.2 del género masculino, con una edad promedio de 20.4 años. La mayoría de los estudiantes mencionaron estar matriculados en carreras como medicina, enfermería, farmacia, lenguas, ingenierías, derecho, turismo, mercadotecnia y relaciones internacionales. En cuanto a su empleabilidad, el $28.8 \%$ indicó que estudiaba y trabajaba, mientras que el $71.2 \%$ solo estudiaba al momento de la investigación. Otros datos importantes de la muestra fueron que el $91.7 \%$ se conecta a internet mayormente desde casa y que el $57.2 \%$ usa el celular para conectarse, seguido por el $33.5 \%$ que utiliza laptop y solo el $8.3 \%$ lo hace desde su computadora de escritorio. Finalmente, en cuanto a la capacitación en el uso de herramientas TIC en los dos últimos años, el $36.3 \%$ señaló que había recibido cursos y talleres, siendo un $63.7 \%$ que indicó cero capacitaciones.

\subsection{Instrumentos y procedimientos}

Se diseñó el Cuestionario de Competencias Digitales del Estudiantado Universitario (Versión 2020), el cual se basó en el Marco Común de Competencia Digital Docente (octubre, 2017). Como se ha mencionado, este marco consta de cinco grandes áreas competenciales que agrupan 21 competencias digitales básicas. Para la redacción de los reactivos del cuestionario se revisaron minuciosamente las descripciones generales de cada una de las cinco áreas: (1) información y alfabetización informacional, (2) comunicación y colaboración, (3) creación de contenidos, (4) seguridad y (5) resolución de problemas. A su vez, se revisaron exhaustivamente las competencias clave de cada área competencial que en total sumaron las 21 competencias digitales. A continuación, se ejemplifica cómo se llegó a la competencia digital integrada en un solo reactivo, el cual se fundamenta 
jerárquicamente en el área competencial 1, en la competencia clave 1.1 hasta dar cabida al reactivo integrador 1.1.1.

\section{Área de competencia 1: Información y alfabetización informacional}

Descripción general: ser capaz de identificar, localizar, obtener, almacenar, organizar y analizar información digital, evaluando su finalidad y relevancia.

\section{Competencia clave:}

1.1. Navegación, búsqueda y filtrado de información, datos y contenidos digitales

Ser capaz de:

- buscar información, datos y contenidos digitales en red, y acceder a ellos,

- expresar de manera organizada las necesidades de información,

- encontrar información relevante para las tareas escolares,

- seleccionar recursos de aprendizaje de forma eficaz,

- manejar distintas fuentes de información,

- crear formas personales de información.

En mi caso particular:

1.1.1. Puedo realizar con éxito la búsqueda de información en la red, filtrando y seleccionando la información, datos y contenido digital; es decir, tomando lo que realmente necesito.

La validación de los 21 reactivos en términos de redacción, claridad y concisión fue llevada a cabo por tres expertos en contenido. Se les explicó la metodología empleada para arribar a las 21 competencias específicas, las cuales tenían que cumplir con la integración de todas las habilidades establecidas en el marco común. Esta tarea cumplió con su propósito y, una vez recibidas y atendidas las observaciones de los expertos de contenido, se procedió a la puesta en línea del instrumento.

En la Figura 2.1 se muestra la versión final del reactivo incorporado a Microsoft Forms. De esta manera, el estudiante puede ver el área competencias y su descripción general, seguido por el reactivo con seis estrellas para indicar su grado de desarrollo. Las estrellas reflejan los seis niveles de competencia establecidos en el Marco Europeo (A1, A2, B1, B2, C1 y C2).

Figura 2

Reactivo muestra del cuestionario en línea

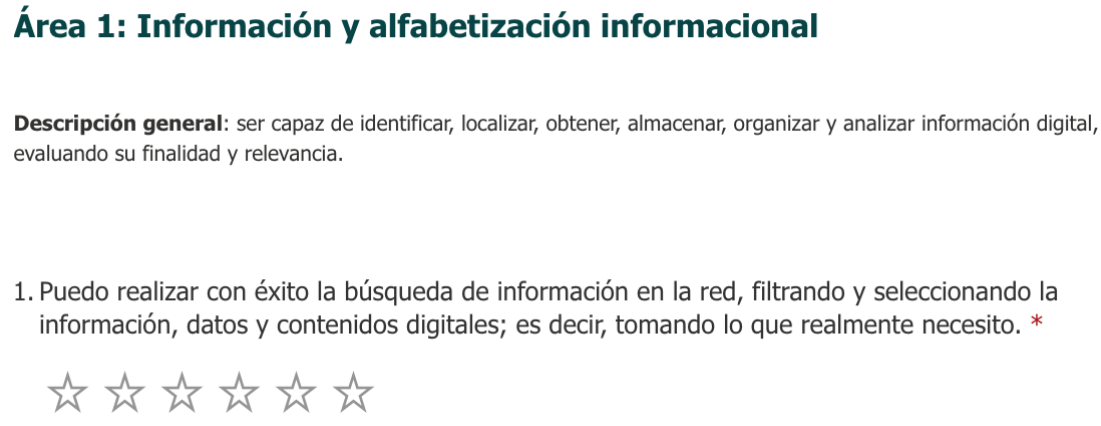

1. Puedo realizar con éxito la búsqueda de información en la red, filtrando y seleccionando la información, datos y contenidos digitales; es decir, tomando lo que realmente necesito. *$$
\text { 论论败论败败 }
$$

Fuente: Elaboración propia 
El segundo instrumento utilizado fue una adaptación del Cuestionario de Personalidad de H. J. Eysenck y S. B. Eysenck (1991) en su versión abreviada, del cual se extrajeron y adaptaron 10 preguntas dicotómicas relacionadas con la introversión-extroversión de los estudiantes. En este breve test se les pide a los participantes que respondan las 10 preguntas contestado Sí o No y sin pensarlo mucho. Por ejemplo:

¿Te gusta platicar mucho?

¿Te consideras una persona animada
Sí No

Sí No

El tercer instrumento fuel el Inventario de Estilos de Aprendizaje D. Kolb y A. Kolb (2013), el cual busca describir las formas de percepción y procesamiento de las cosas dando luz a cuatro grandes estilos de aprendizaje: (1) divergente (2) asimilador, (3) convergente y (4) acomodador. El inventario consta de 12 reactivos con cuatro tipos de preferencias de aprendizaje; es decir, los usuarios responden a cuatro situaciones breves seleccionando 4 en las opciones que más los describen, 1 en las que menos, 3 y 2 como puntos intermedios. Por ejemplo:

Cuando aprendo:

Prefiero valerme de mis sentimientos y sensaciones.

Prefiero mirar y atender.

Prefiero pensar con mis ideas.

Prefiero hacer cosas

Estos tres instrumentos de medición fueron integrados en un solo cuestionario por razones prácticas en la recolección de datos en tiempos de pandemia por el COVD-19. Fueron 43 los reactivos incorporados al instrumento que empiezan con las 21 competencias digitales básicas, los 10 reactivos de introversiónextroversión, los 12 reactivos sobre los estilos de aprendizaje. De igual forma, se agregó una sección para recabar la información demográfica de los participantes; por ejemplo, el género, la edad, la carrera que cursa, la institución educativa, la empleabilidad, las herramientas tecnológicas, la capacitación, entre otras. El instrumento integrado se aplicó en línea a través de los profesores que se encontraban impartiendo clases y por medio de invitaciones directas a los interesados. En promedio, el cuestionario fue contestado en 12.5 minutos y mayormente con el celular.

Cabe señalar que se agregó, en el cuestionario, un reactivo para que los participantes escribieran un correo electrónico válido por si deseaban recibir su resultado personalizado sobre el grado de desarrollo de sus competencias digitales. En este sentido, el 65.4 \% de los estudiantes ingresó un correo electrónico, el cual refleja el interés en saber sus resultados.

\subsection{Análisis de los datos}

Los datos recolectados a través de Microsoft Forms fueron descargados en un archivo de Excel y adecuados para ser exportados al paquete estadístico IBM SPSS Versión 25. Las 21 competencias digitales tuvieron una escala del 1 al 6 reflejando los 6 niveles propuestos en el Marco Común de Competencia Digital Docente, los cuales fueron reducidos a tres: principiante, intermedio y avanzado. Los 12 reactivos del Inventario de Estilos de Aprendizaje fueron contabilizados en términos de percepción (experiencia concreta y conceptualización abstracta) y de procesamientos (experimentación activa y observación reflexiva), cuyas combinaciones dieron cabida a los cuatro estilos de aprendizaje de Kolb (2013): divergente, asimilador, convergente y acomodador. Por otro lado, los 10 reactivos de extroversión, se registraron como la suma total sobre un continuo de 0 (introversión) a 10 (extroversión). De esta manera, los datos de personalidad pudieron analizarse como escalas categóricas o numéricas. 
Para contestar la pregunta sobre las competencias reportadas con mayor y menor desarrollo, se tuvieron en cuenta las medias de frecuencia de las 21 competencias, así como las medias de las cinco áreas competenciales; es decir, estadísticos descriptivos. Para el resto de las preguntas de investigación se utilizaron estadísticas inferenciales tales como la correlación de Pearson y la ANOVA de un factor.

\section{Resultados}

Una vez analizados los datos y realizadas las pruebas estadísticas, se presentan los resultados de este estudio siguiendo el orden en que las cuatro preguntas de investigación fueron formuladas. En la siguiente sección se discutirán los resultados de manera concisa y puntual.

\subsection{Las competencias digitales con mayor y menor desarrollo}

Con base en la Tabla 1, se puede sugerir que los participantes tienen un nivel autopercibido medio alto en las 21 competencias digitales ( 4.8 global). La medias más bajas estuvieron entre 3.78 y 4.63 , mientras que las medias más altas se dieron entre 5 y 5.41 . Se puede decir que entre las competencias con mayor desarrollo fueron: la comunicación por medios digitales, la gestión de la información y de la propia identidad, la aplicación de la Netiqueta, así como el cuidado del entorno personal y del medio ambiente.

Tabla 1

Las 21 competencias digitales organizadas por su media

\begin{tabular}{|l|r|r|r|}
\hline Competencia digital & N & Media & Desv. Est. \\
\hline 4. Comunicarse mediante las tecnologías digitales. & 278 & $\mathbf{5 . 4 1}$ & .882 \\
\hline 1. Buscar, filtrar y seleccionar información, datos y contenidos digitales. & 278 & $\mathbf{5 . 2 8}$ & .926 \\
\hline 5. Compartir información y contenidos digitales. & 278 & $\mathbf{5 . 2 7}$ & .990 \\
\hline 9. Gestionar la propia identidad y reputación digitales. & $\mathbf{2 7 8}$ & $\mathbf{5 . 2 0}$ & 1.028 \\
\hline 8. Aplicar las normas sociales para la sana convivencia (Netiqueta). & 278 & $\mathbf{5 . 1 8}$ & 1.072 \\
\hline 17. Proteger el entorno personal y para el cuidado del medio ambiente. & 278 & $\mathbf{5 . 0 0}$ & 1.056 \\
\hline 2. Evaluar o valorar la información, datos y contenidos digitales. & 278 & 4.99 & 1.002 \\
\hline 3. Almacenar la información, datos y contenidos digitales en la "nube". & 278 & 4.94 & 1.269 \\
\hline $\begin{array}{l}\text { 6. Colaborar en redes ciudadanas o escolares para mejorar entorno social y } \\
\text { educativo. }\end{array}$ & 278 & 4.91 & 1.138 \\
\hline $\begin{array}{l}\text { 10. Crear contenidos digitales (elaboración propia) haciendo uso de los diferentes } \\
\text { recursos. }\end{array}$ & 278 & 4.88 & 1.143 \\
\hline 16. Proteger la salud por el uso excesivo de los dispositivos. & 278 & 4.87 & 1.161 \\
\hline 21. Mejorar la competencia digital tomando cursos o siendo autodidacta. & 278 & 4.78 & 1.224 \\
\hline 11. Integrar y adaptar contenidos digitales creados por otros (reelaboración). & 278 & 4.75 & 1.234 \\
\hline $\begin{array}{l}\text { 19. Identificar las limitaciones en el uso de la tecnología para solucionar } \\
\text { problemas técnicos. }\end{array}$ & 278 & 4.74 & 1.231 \\
\hline 7. Participar en diferentes canales digitales (foros de discusión y temáticos). & 278 & 4.69 & 1.188 \\
\hline \begin{tabular}{l} 
15. Proteger los datos personales e identidad digital en la red. \\
\hline 18. Resolver problemas técnicos básicos en los dispositivos.
\end{tabular} & 278 & 4.69 & 1.293 \\
\hline 20. Innovar utilizando la tecnología digital para resolver problemas técnicos. & 278 & $\mathbf{4 . 6 3}$ & 1.387 \\
\hline
\end{tabular}




\begin{tabular}{|c|c|c|c|}
\hline Competencia digital & $\mathrm{N}$ & Media & Desv. Est. \\
\hline $\begin{array}{l}\text { 14. Proteger los dispositivos y contenidos digitales propios de amenazas de virus o } \\
\text { hackeo. }\end{array}$ & 278 & 4.31 & 1.488 \\
\hline 12. Aplicar los diferentes tipos de derechos de autor y licencias. & 278 & 4.25 & 1.432 \\
\hline 13. Hacer modificaciones básicas a algún software o aplicaciones (programación). & 278 & 3.78 & 1.706 \\
\hline N válido (por lista) & 278 & & \\
\hline
\end{tabular}

Fuente: Elaboración propia

Por otro lado, entre las competencias autopercibidas con menor desarrollo se encontraron la programación básica, la aplicación de licencias y derechos de autor, la protección de dispositivos, la innovación para resolver problemas técnicos en los dispositivos.

La Tabla 2 muestra las cinco áreas competenciales ordenadas con sus medias de manera descendente. De esta forma, se puede mencionar que las categorías de comunicación y colaboración e información y alfabetización informacional resultaron como la más desarrolladas, con medias de 5.11 y 5.06 cada una.

Tabla 2

Las 21 competencias digitales organizadas por medias descendentes

\begin{tabular}{|c|c|c|c|}
\hline Competencias digitales por área & $\mathrm{N}$ & Media & Desv. Est. \\
\hline Comunicación y colaboración & 278 & 5.1109 & .84189 \\
\hline Información y alfabetización digital & 278 & 5.0695 & .86745 \\
\hline Seguridad & 278 & 4.7185 & 1.05389 \\
\hline Resolución de problemas & 278 & 4.6691 & 1.12114 \\
\hline Creación de contenidos digitales & 278 & 4.4137 & 1.14622 \\
\hline $\mathrm{N}$ válido (por lista) & 278 & & \\
\hline
\end{tabular}

Fuente: Elaboración propia

En contraste, el área competencias menos desarrollada fue la creación de contenidos digitales. Esta área engloba la elaboración propia de materiales, la adaptación de materiales existente, la aplicación de licencias y derechos de autor, así como la programación en un nivel básico.

\subsection{Personalidad y competencias digitales}

Como se ha mencionado, el continuo de introversión-extroversión tuvo una escala del 0 al 10. La Figura 3 muestra la distribución de los 278 casos a lo largo de este continuo con una media de 5.51 y una desviación estándar de 2.89 .

Asimismo, una mirada a la distribución de la variable introversión-extroversión puede sugerir al menos tres grupos: introvertidos (con puntuaciones entre 0 y 3); intermedios (entre 4 y 7 ) y extrovertidos (entre 8 y 10). En la Tabla 3.3 se visualiza la distribución de esta variable en el que el 19.4 de la muestra resultó tendencia hacia la introversión y el 31.7 \% hacia la extroversión. En un punto intermedio, se observa que el 48.9 \% tiene una orientación balanceada. 
Figura 3

Distribución de los puntajes de extroversión

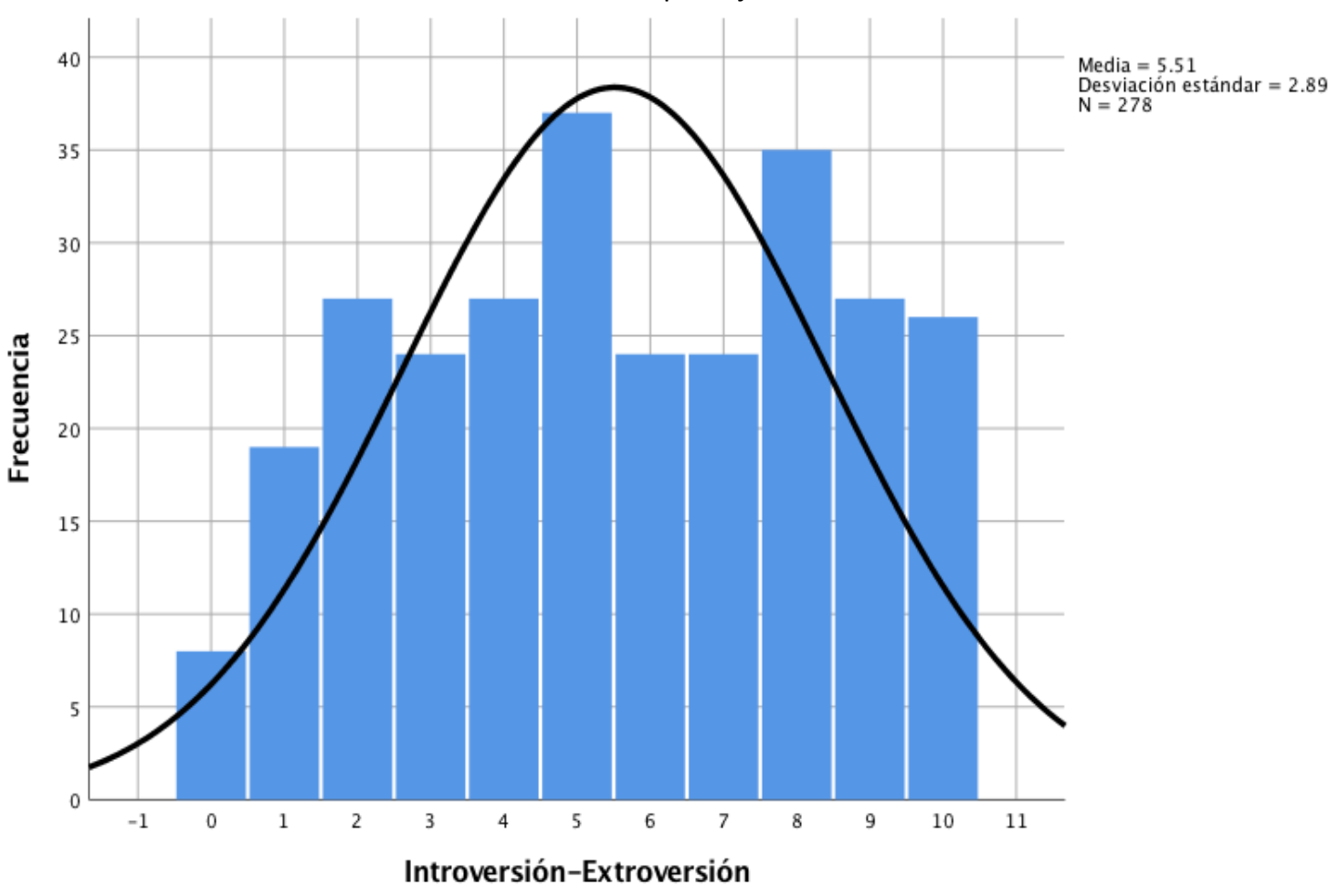

Fuente: Elaboración propia

Tabla 3

Distribución de extroversión en tres grupos

\begin{tabular}{|l|l|c|c|c|c|}
\hline \multicolumn{2}{|c|}{} & Frecuencia & Porcentaje & Porcentaje válido & Porcentaje acumulado \\
\hline \multirow{4}{*}{ Válido } & Introvertidos & 54 & 19.4 & 19.4 & 19.4 \\
\cline { 2 - 6 } & Intermedios & 136 & 48.9 & 48.9 & 68.3 \\
\cline { 2 - 6 } & Extrovertidos & 88 & 31.7 & 31.7 & 100.0 \\
\cline { 2 - 6 } & Total & 278 & 100.0 & 100.0 & \\
\hline
\end{tabular}

Fuente: Elaboración propia

Independiente del tratamiento de la escala numérica o nominal de la variable introversión-extroversión, se encontró que de las 21 competencias digitales específicas, únicamente hubo una baja correlación positiva en tres de ellas. En la Tabla 4 se presentan las relaciones positivas entre extroversión y la medida en que los estudiantes participan en diferentes canales digitales, hacen modificaciones básicas a algún software o aplicación y protegen sus datos personales e identidad digital en la red.

De igual forma, se encontraron correlaciones débiles pero estadísticamente significativas entre la extroversión, como factor de personalidad, y dos de las cinco áreas o categorías competenciales establecidas en el Marco Común antes mencionado. 
Tabla 4

Correlaciones de extroversión con competencias digitales específicas

\begin{tabular}{|c|c|c|}
\hline Competencia digital & & $\mathrm{R}$ \\
\hline \multirow[t]{3}{*}{ 7. Participar en diferentes canales digitales (foros de discusión y temáticos). } & Correlación de Pearson & $.130^{*}$ \\
\hline & Sig. (bilateral) & .030 \\
\hline & $\mathrm{N}$ & 278 \\
\hline \multirow{3}{*}{$\begin{array}{l}\text { 13. Hacer modificaciones básicas a algún software o aplicaciones } \\
\text { (programación). }\end{array}$} & Correlación de Pearson & $.128^{*}$ \\
\hline & Sig. (bilateral) & .033 \\
\hline & $\mathrm{N}$ & 278 \\
\hline \multirow[t]{3}{*}{ 15. Proteger los datos personales e identidad digital en la red. } & Correlación de Pearson & $.147^{*}$ \\
\hline & Sig. (bilateral) & .014 \\
\hline & $\mathrm{N}$ & 278 \\
\hline
\end{tabular}

Fuente: Elaboración propia

De esta manera, en la Tabla 5 se observan las correlaciones positivas entre extroversión y el área de creación de contenidos digitales y el área de seguridad. En resumen, estos resultados parecen indicar que a mayor grado de extroversión pueden haber un mayor grado de desarrollos de las tres competencias específicas y las dos áreas competenciales. Dicho de otro modo, si se tratase de dos grupo de introvertidos y extrovertidos, serían los extrovertidos los que se autopercibieran con mayores habilidades en estas competencias.

Tabla 5

Correlación de extroversión y competencias digitales por área

\begin{tabular}{|l|l|c|c|c|c|c|}
\hline \multicolumn{2}{|c|}{} & $\begin{array}{c}\text { Información y } \\
\text { alfabetización } \\
\text { informacional }\end{array}$ & $\begin{array}{c}\text { Comunicación } \\
\text { y colaboración }\end{array}$ & $\begin{array}{c}\text { Creación de } \\
\text { contenidos } \\
\text { digitales }\end{array}$ & $\begin{array}{c}\text { Resolución } \\
\text { de } \\
\text { Seguridad } \\
\text { problemas }\end{array}$ \\
\hline $\begin{array}{l}\text { Introversión- } \\
\text { Extroversión }\end{array}$ & Correlación de Pearson & .055 & .089 & $.125^{*}$ & $.137^{*}$ & .063 \\
\cline { 2 - 7 } & Sig. (bilateral) & .358 & .138 & .037 & .022 & .295 \\
\cline { 2 - 8 } & $\mathrm{N}$ & 278 & 278 & 278 & 278 & 278 \\
\hline \multirow{2}{*}{$*$ La correlación es significativa en el nivel 0,05 (bilateral). }
\end{tabular}

Fuente: Elaboración propia

\subsection{Estilos de aprendizaje y competencias digitales}

Para efectos de resultados, la variable estilos de aprendizaje contemplo cuatro niveles: divergente, asimilador, convergente y acomodador. Estos cuatro estilos se derivan de las combinaciones entre los modos de percepción (experimentación concreta y conceptualización abstracta) y los modos de procesamiento (experimentación activa y observación reflexiva). La Tabla 6 muestra la distribución de los cuatro estilos en una muestra aleatoria de 152 estudiantes.

Tabla 6

Distribución de los estilos de aprendizaje de la muestra

\begin{tabular}{|l|l|c|c|c|c|}
\hline \multicolumn{2}{|c|}{} & Frecuencia & Porcentaje & Porcentaje válido & Porcentaje acumulado \\
\hline \multirow{4}{*}{ Válido } & Asimilador & 63 & 22.7 & 36.8 & 36.8 \\
\cline { 2 - 6 } & Convergente & 40 & 14.4 & 23.4 & 60.2 \\
\cline { 2 - 6 } & Acomodador & 31 & 11.2 & 18.1 & 78.4 \\
\cline { 2 - 6 } & Divergente & 37 & 13.3 & 21.6 & 100.0 \\
\cline { 2 - 6 } & Total & 171 & 61.5 & 100.0 & \\
\hline
\end{tabular}

Fuente: Elaboración propia. 
Como se puede observar, el $36.8 \%$ de los estudiantes tiende a desarrollar un estilo de aprendizaje asimilador, mientras que el $18.1 \%$ parece orientado al estilo acomodador. El segundo estilo de aprendizaje con mayor porcentaje fue el convergente con un 23.4, seguido muy de cerca por el estilo divergente con un $21.6 \%$.

En cuando a posibles diferencias estadísticamente significativas entre los cuatro estilos de aprendizaje y el desarrollo de las competencias digitales, aparentemente los estilos de aprendizaje no explican tales diferencias; esto de acuerdo con la prueba de ANOVA de un factor. Sin embargo, un análisis descriptivo teniendo en cuenta tres niveles de competencia cruzados con los cuatro estilos, proporciona un panorama interesante. En la Tabla 7 se aprecia la distribución de los cuatro estilos por los niveles de competencia en el área de Información y Alfabetización Informacional.

Tabla 7

Estilos de aprendizaje e información y alfabetización informacional

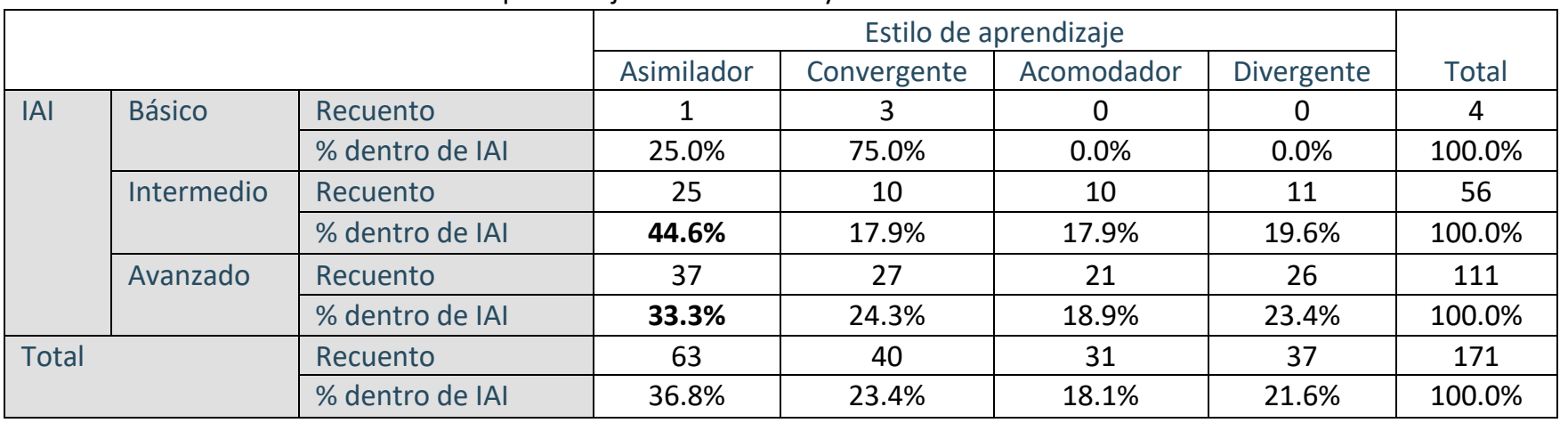

Fuente: Elaboración propia.

En esta área competencial, los estudiantes asimiladores parecen distinguirse de los demás en los niveles intermedio y avanzado, con $44.6 \%$ y $33.3 \%$ respectivamente. Es decir, de 111 estudiantes avanzados, 37 resultaron ser asimiladores. De igual manera, de 56 estudiantes intermedios, 25 también fueron asimiladores.

En cuanto al área competencias de Comunicación y Colaboración (véase Tabla 8), nuevamente los estudiantes con orientación de asimilador parecen sobresalir más en los niveles intermedio y avanzado. Es decir, el $50 \%$ de los estudiantes intermedios y el $34.5 \%$ de los estudiantes avanzados resultaron asimiladores.

Tabla 8

Estilos de aprendizaje y comunicación/colaboración

\begin{tabular}{|c|c|c|c|c|c|c|c|}
\hline & \multicolumn{4}{|c|}{ Estilo de aprendizaje } & \multirow[b]{2}{*}{ Total } \\
\hline & & & Asimilador & Convergente & Acomodador & Divergente & \\
\hline \multirow[t]{6}{*}{ CyC } & \multirow[t]{2}{*}{ Básico } & Recuento & 2 & 5 & 3 & 4 & 14 \\
\hline & & $\%$ dentro de CyC & $14.3 \%$ & $35.7 \%$ & $21.4 \%$ & $28.6 \%$ & $100.0 \%$ \\
\hline & \multirow[t]{2}{*}{ Intermedio } & Recuento & 22 & 7 & 5 & 10 & 44 \\
\hline & & $\%$ dentro de CyC & $50.0 \%$ & $15.9 \%$ & $11.4 \%$ & $22.7 \%$ & $100.0 \%$ \\
\hline & \multirow[t]{2}{*}{ Avanzado } & Recuento & 39 & 28 & 23 & 23 & 113 \\
\hline & & $\%$ dentro de CyC & $34.5 \%$ & $24.8 \%$ & $20.4 \%$ & $20.4 \%$ & $100.0 \%$ \\
\hline \multirow{2}{*}{\multicolumn{2}{|c|}{ Total }} & Recuento & 63 & 40 & 31 & 37 & 171 \\
\hline & & $\%$ dentro de CyC & $36.8 \%$ & $23.4 \%$ & $18.1 \%$ & $21.6 \%$ & $100.0 \%$ \\
\hline
\end{tabular}

Fuente: Elaboración propia.

En esta área competencial, también sobresalen los estudiantes orientados al estilo convergente con un $35.7 \%$ en el nivel básico, aunque no muy alejados numéricamente de los divergentes. De hecho, independientemente del estilo de aprendizaje, solo el 8.1 \% de la muestra se autopercibió con un nivel competencial básico. 
La tercera área competencial fue la de Creación de Contenidos Digitales como se aprecia en la Tabla 9. En esta ocasión los asimiladores sobresalieron en los tres niveles de competencia, básico, intermedio y avanzado (38.1 $\%, 38.3 \%$ y $34.8 \%)$.

Tabla 9

Estilos de aprendizaje y creación de contenidos digitales

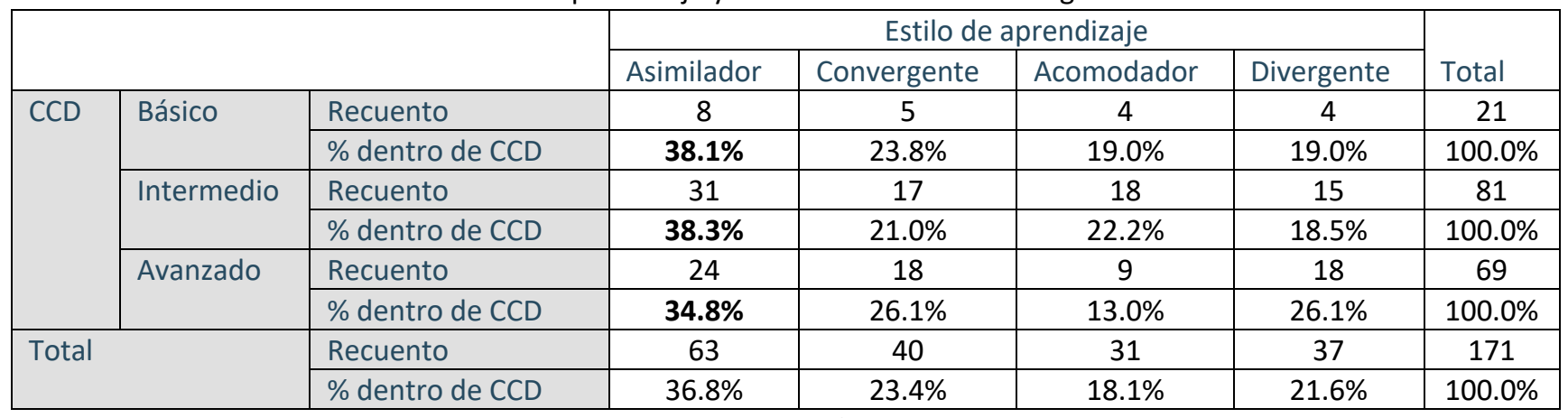

Fuente: Elaboración propia.

Como se puede notar en la Tabla 9, los otros estilos de aprendizaje obtuvieron procentajes similares; por ejemplo, convergentes, acomodadores y divergentes en los niveles básico e intermedio. En el nivel avanzado, los porcentajes entre convergentes y los divergentes fueron iguales.

Con respecto a la cuarta área competencial de Seguridad, la Tabla 10 muestra resultados parecidos al área de Información y Alfabetización Informacional. Los porcentajes mayores en los niveles intermedio y avanzado fueron cubiertos por los estudiantes asimiladores ( $40 \%$ y $35.4 \%$ ). En el nivel básico, aunado a que hubo muy pocos casos, ningún estilo pareció sobresalir.

Tabla 10

Estilos de aprendizaje y seguridad

\begin{tabular}{|c|c|c|c|c|c|c|c|}
\hline & & & \multicolumn{4}{|c|}{ Estilo de aprendizaje } & \multirow[t]{2}{*}{ Total } \\
\hline & & & Asimilador & Convergente & Acomodador & Divergente & \\
\hline \multirow[t]{6}{*}{ SEG } & \multirow[t]{2}{*}{ Básico } & Recuento & 2 & 3 & 1 & 3 & 9 \\
\hline & & $\%$ dentro de SEG & $22.2 \%$ & $33.3 \%$ & $11.1 \%$ & $33.3 \%$ & $100.0 \%$ \\
\hline & \multirow[t]{2}{*}{ Intermedio } & Recuento & 32 & 19 & 15 & 14 & 80 \\
\hline & & $\%$ dentro de SEG & $40.0 \%$ & $23.8 \%$ & $18.8 \%$ & $17.5 \%$ & $100.0 \%$ \\
\hline & \multirow[t]{2}{*}{ Avanzado } & Recuento & 29 & 18 & 15 & 20 & 82 \\
\hline & & $\%$ dentro de SEG & $35.4 \%$ & $22.0 \%$ & $18.3 \%$ & $24.4 \%$ & $100.0 \%$ \\
\hline \multirow{2}{*}{\multicolumn{2}{|c|}{ Total }} & Recuento & 63 & 40 & 31 & 37 & 171 \\
\hline & & $\%$ dentro de SEG & $36.8 \%$ & $23.4 \%$ & $18.1 \%$ & $21.6 \%$ & $100.0 \%$ \\
\hline
\end{tabular}

Fuente: Elaboración propia.

Finalmente, en el área competencial de Resolución de Problemas, una vez más los estudiantes asimiladores resultaron con mayores proporciones en los niveles intermedio y avanzado ( $42.3 \%$ y $34.9 \%$ ), como se observa en la Tabla 11. Al igual que en el área de Seguridad, la distribución de los pocos casos en el nivel básico fue equitativa para los cuatro estilos de aprendizaje. 
Tabla 11

Estilos de aprendizaje y resolución de problemas

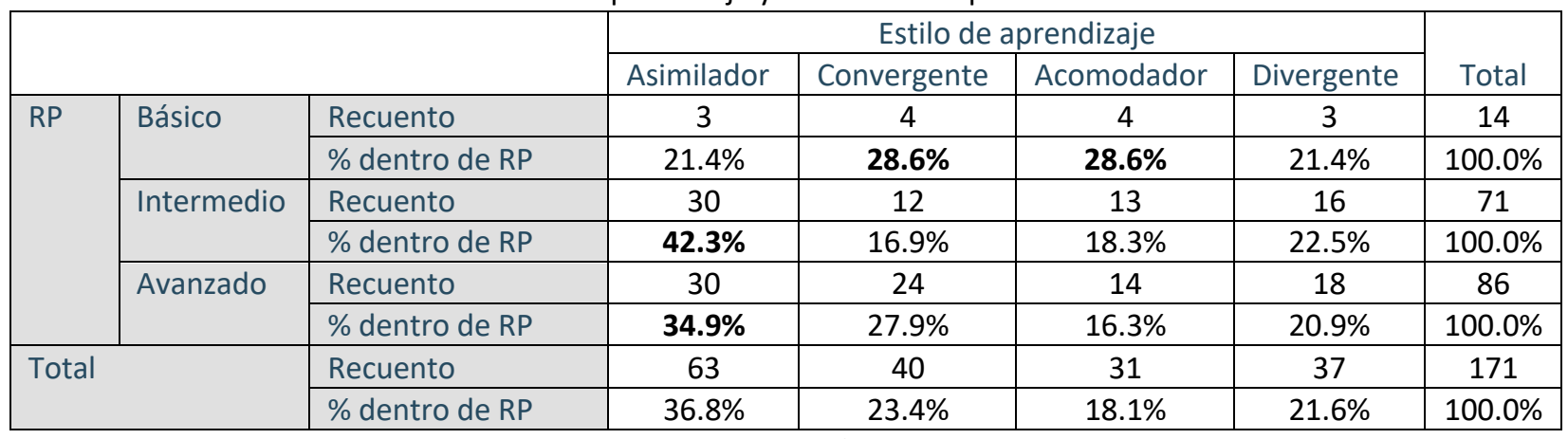

Fuente: Elaboración propia.

En resumen, en este breve análisis entre las variables de estilos de aprendizaje y los niveles de competencia digital en sus cinco áreas, los estudiantes con estilo asimilador sobresalieron en las áreas competenciales de Información y Alfabetización Informacional, Comunicación y Colaboración, Creación de Contenidos Digitales, Seguridad y Resolución de Problemas. La proporción mayor se dio nivel intermedio más que en el avanzado. Fue en el nivel básico, con un número menor de casos en total, en el que la proporción de los cuatro estilos de aprendizaje fue similar.

\subsection{Discusión}

El grado de desarrollo autopercibido de las 21 competencias digitales de los 278 estudiantes universitarios tuvo un rango entre intermedio y avanzado, con una media mínima de 3.78 y una máxima de 5.41. Quizá las autovaloraciones altas pudieran estar derivadas de la contingencia sanitaria en la que los estudiantes se vieron en la necesidad de utilizar con mayor frecuencia los recursos tecnológicos para fines educativos. Esto pudo haber tenido un impacto positivo en sus competencias digitales. En cuanto a las competencias con mayor y menor desarrollo, los resultados coinciden en gran medida con los encontrados en Marín, Hernández, Borges y Blanqueto (2021), cuyos datos fueron levantados antes de la pandemia. Es decir, las competencias digitales relacionadas con la comunicación estuvieron entre las más altas y las vinculadas a la creación de contenidos entre las más bajas. Estos resultados también parecen alinearse con los reportados por Guzmán Flores (2008), Guzmán Flores y Gisbert Cervera (2008), Cabero et al (2009), Vázquez-Cano, Reyes Vélez, Colmenares Zamora, y López Meneses (2017) y Sánchez-Macías y Veytia-Bucheli (2019).

Por otro lado, la introversión-extroversión, como factor de personalidad parece estar poco relacionada con el grado de desarrollo de las competencias digitales del estudiantado. Si bien es cierto que las correlaciones fueron débiles, se pudo observar una tendencia a favor de los estudiantes más extrovertidos; sobre todo, en las áreas competenciales de creación de contenidos y de seguridad. Esto pudiera explicarse que los extrovertidos, por sus características personológicas, tiendan a elaborar o adaptar contenidos digitales utilizando una variedad de recursos tecnológicos que los motive también a desarrollar competencias relacionadas con la seguridad. Aún cuando la literatura es muy escasa, estos resultados son congruentes con los estudios de Nahyun y Hana (2011) y Ahmed y Rasheed (2020), quienes encontraron una relación positiva entre la extroversión y ciertas formas de competencias digitales.

Con respecto a los estilos de aprendizaje y su relación con las competencias digitales, los resultados no fueron estadísticamente significativos, resultado similar al de Orellana, Bo, Belloch y Aliaga (2002). Pareciera que los estudiantes asimiladores, convergentes, acomodadores y divergentes se autopercibieron con medias similares en su desarrollo competencial. Una posibilidad de este resultado podría también estar vinculado a la contingencia sanitaria bajo el supuesto de que los estudiantes no tuvieron opción para cumplir con los 
requerimientos y actividades de los cursos en línea. No obstante, el análisis por los tres niveles de competencias y los cuatro estilos sugirió que fueron más los asimiladores quienes se autopercibieron en los niveles intermedio y avanzado. Esto resultados parciales resultan interesantes dado que se muestra cierta variabilidad y preponderancia hacia un estilo de aprendizaje como en el caso de Romero, Salinas y Mortera (2010, quienes adujeron que la Plataforma Moodle privilegia el estilo convergente. También está lo reportado por Chapilliquén (2015) en cuya investigación los estilos predominantes y su desempeño en competencias digitales fueron los estudiantes sensoriales y visuales de un grupo experimental.

\section{Conclusiones}

Ciertamente, la relación entre la personalidad y los estilos de aprendizaje con las competencias digitales ha sido poco estudiada. Los resultados de esta investigación, en esta línea, han sido parciales pero relevantes. Por lo tanto, se requiere continuar explorando estas variables de tipo personal que pudieran arrojar hallazgos más consistentes. Para esto, es necesario utilizar otros instrumentos para medir la personalidad y los estilos de aprendizaje que fueran más sensibles a las competencias digitales. También se pueden recurrir a otros diseños de investigación como los de corte cualitativo y experimental dado que los estudios basados en encuestas tienen sus limitaciones.

Por otro lado, las implicaciones pedagógicas de los principales hallazgos sí pueden sugerir el tener en cuenta los rasgos de personalidad y los diferentes estilos de aprendizaje no solo en el diseño de recursos digitales sino también en las formas de instrucción y evaluación del desempeño de los estudiantes. Siempre será importante conocer las características y las preferencias de los estudiantes para la mejora continua en el ámbito educativo, sobre todo en tiempos de la nueva realidad derivada de la contingencia sanitaria.

\section{Referencias bibliográficas}

Ahmed, S. y Rasheed, T. (2020). Relationship between personality traits and digital literacy skills: a study of university librarians, Digital Library Perspectives, Vol. 36 No. 2, pp. 191-206. https://doi.org/10.1108/DLP02-2020-0005

Cabero, J., Llorente, M., Leal, F., y Andrés, F. (2009) La alfabetización digital de los alumnos universitarios mexicanos: una investigación en la Universidad Autónoma de Tamaulipas. Enseñanza and Teaching. 27 (1), pp. 41-59. https://gredos.usal.es/handle/10366/79276

Chapilliquén Rodríguez, M. (2015). Competencias digitales en estudiantes con diferentes estilos de aprendizaje del séptimo ciclo de educación secundaria, desarrolladas a través de la red social educativa Edmodo en una institución educativa pública de la Unidad de Gestión Educativa Local No. 03. Tesis de Maestría. Pontificia Universidad Católica del Perú. http://tesis.pucp.edu.pe/repositorio/handle/20.500.12404/6744

De Moya, M., Hernández, J., Hernández, J. y Cózar, R. (2011). Análisis de los estilos de aprendizaje y las TIC en la formación personal del alumnado universitario a traves del cuestionario REATIC. Revista de Investigación Educativa, 29 (1), 137-156. https://www.redalyc.org/articulo.oa?id=283322813008

Eysenck, H.J. (1947) Dimensions of Personality. London: Methuen.

Eysenck, H. J. (1967). The biological Basis of personality. Springfield, IL: Charles C. Thomas.

Eysenck, H. J. (1990). Biological dimensions of personality. En L. A. Pervin (Ed.), Handbook of personality: Theory and research (pp. 244-276). New York: Guilford. 
Eysenck, H. J., and Eysenck, S. B. (1991) The Eysenck Personality Questionnaire-Revised. London: Hodder and Stoughton.

Eysenck, H. J. and Eysenck, S. B. (1969) Personality Structure and Measurement. London: Routledge \& Kegan Paul.

Guzmán Flores, T. (2008). Las tecnologías de la información y la comunicación en la Universidad Autónoma de Querétaro: propuesta estratégica para su integración. Tesis doctoral. Universitat Rovira i Virgili, http://www.tdx.cat/handle/10803/8937

Guzmán Flores, T. y Gisbert Cervera, M. (2008). Competencias TIC de los estudiantes de la Universidad Autónoma de Querétaro (UAQ): Un estudio de caso. Comunicación presentada en Virtual Educa. Portal Educativo de las Américas Organización de los Estados Americanos https://recursos.educoas.org/sites/default/files/12631.pdf

INTEF (2017) Marco Común de Competencia Digital Docente. Ministerio de Educación, Cultura y Deporte de España. https://aprende.intef.es/sites/default/files/2018-05/2017_1020_Marco-Común-de-CompetenciaDigital-Docente.pdf

Keefe, J.W. (1979). Learning style: An overview. En J. W. Keefe (Ed.), Student learning styles: Diagnosing and prescribing programs (pp. 1-17). Reston, VA: National Association of Secondary School Principals. https://archive.org/details/studentlearnings0000unse_y0p6

Kolb, D. (1984). Experiential learning: Experience as the source of learning and development. New Jersey: Prentice-Hall.

Kolb, D. y Kolb, A. (2013) The Kolb Learning Style Inventory 4.0. A comprehensive guide to theory, psychometrics, research and applications. Upper Saddle River, New Jersey: Experience Based Learning Systems. https://www.researchgate.net/publication/303446688_The_Kolb_Learning_Style_Inventory_40_Guide_to _Theory_Psychometrics_Research_Applications

Kolb, D. (2015) Experiential learning. Experience as the source of learning and development. Upper Saddle River, New Jersey: Experience Based learning Systems, Inc.

Marín-Marín, A. (2005). Extraversion and the use of vocabulary learning strategies among university EFL students in Mexico. Tesis doctoral no publicada. Universidad de Essex. RU.

Marin-Marin, A., Hernández-Romero, M. I., Borges-Ucán, J. L., y Blanqueto-Estrada, M. (2021). La Competencia digital del estudiantado universitario. Revista Transdigital, 2(3). https://www.revistatransdigital.org/index.php/transdigital/article/view/48

Nahyun, K., y Hana, S. (2011). Personality traits, gender, and information competency among college students. Malaysian Journal \& Information Science. 16 (1), 87-107, https://mjlis.um.edu.my/index.php/MJLIS/article/view/6686

Orellana, N., Bo, R., Belloch, C., y Aliaga, F (2002). Estilos de aprendizaje y utilización de las TIC en la enseñanza superior. Presentado en Virtual Educa, España. https://www.researchgate.net/publication/258860151_ESTILOS_DE_APRENDIZAJE_Y_UTILIZACION_DE_L AS_TIC_EN_LA_ENSENANZA_SUPERIOR 
Romero, L., Salinas, U. y Mortera (2010). Estilos de aprendizaje basados en el modelo de Kolb en la educación virtual. Apertura, vol. 2, núm. 1. http://www.udgvirtual.udg.mx/apertura/index.php/apertura/article/view/21/30

Sánchez-Macías, A., y Veytia-Bucheli, M. G. (2019). Las competencias digitales en estudiantes de doctorado. Un estudio en dos universidades mexicanas. Academia y Virtualidad, 12(1), 7-30.

https://doi.org/10.18359/ravi.3618

Vergara, C. (s.f.) La teoría de los estilos de aprendizaje de Kolb. Actualidad en Psicología. https://www.actualidadenpsicologia.com/la-teoria-de-los-estilos-de-aprendizaje-de-kolb/

Vázquez-Cano, E., Reyes Vélez, M., Colmenares Zamora, L., \& López Meneses, E. (2017). Competencia digital del alumnado de la Universidad Católica de Santiago de Guayaquil. Opción, 33(83), 229-251. https://dialnet.unirioja.es/descarga/articulo/6228337.pdf

\section{(cc) EY-NC}

Esta obra está bajo una Licencia Creative Commons Atribución-NoComercial 4.0 Internacional 